\title{
Weakening of the spin density wave gap at low temperatures in $\mathrm{SrFe}_{2} \mathrm{As}_{2}$ single crystals
}

\author{
Anirban Dutta, ${ }^{1, \text { a }}$ Neeraj Kumar, ${ }^{2}$ A. Thamizhavel, ${ }^{2}$ and Anjan K. Gupta ${ }^{1}$ \\ ${ }^{1)}$ Department of Physics, Indian Institute of Technology Kanpur, Kanpur 208016, \\ India \\ ${ }^{2)}$ Department of Condensed Matter Physics and Materials Science, Tata Institute of Fundamental Research, \\ Homi Bhabha Road, Colaba, Mumbai 400 005, India
}

(Dated: 24 September 2018)

\begin{abstract}
We report on temperature dependent scanning tunneling microscopy and spectroscopy studies of undoped $\mathrm{SrFe}_{2} \mathrm{As}_{2}$ single crystals from $6 \mathrm{~K}$ to $292 \mathrm{~K}$. Resistivity data show spin density wave (SDW) transition at $\mathrm{T}_{S D W} \approx 205 \mathrm{~K}$ and the superconducting transition at $\sim 21 \mathrm{~K}$ while magnetic susceptibility does not show any superconductivity. Conductance maps and local tunneling spectra show an electronically homogeneous surface at all studied temperatures. Tunnel spectra correlate well with the SDW transition with a depression in the density of states near the Fermi energy below $\mathrm{T}_{S D W}$. On further lowering the temperature, the width of this partial energy gap associated with the SDW decreases. Using the anti-correlation of superconducting phase with SDW, we interpret this gap weakening as a precursor to superconductivity. This may give rise to a facile nucleation of superconductivity near defects.
\end{abstract}

PACS numbers: 72.27.+a,74.70.Xa,75.30.Fv

The superconductivity (SC) in FeAs based layered compounds ${ }^{1}$, occurs in close proximity with the stripe type anti-ferromagnetic (AFM) phase $^{2-4}$ when the latter is suppressed by chemical doping ${ }^{2}-\underline{4}$ or pressure ${ }^{3,5}$. In this respect, pnictides are similar to the layered cuprates and thus the spin-ordering, common to the two, is suspected to have some role in their superconductivity. Thus probing this interface between magnetic ordering and superconductivity has been of significant interest. The parent compounds of pnictides are bad metals, while parent cuprates are antiferromagnetic Mott insulators. The parent compounds of 122 pnictides $\left(A F e_{2} A s_{2}, \mathrm{~A}: \mathrm{Ca}, \mathrm{Ba}\right.$, $\mathrm{Sr}$ or Eu) undergo a spin density wave (SDW) transition at a temperature $\left(T_{S D W}\right)$ ranging from 125 to 210 $\mathrm{K}$, depending on $\mathrm{A}^{6}-\underline{6}$. Angle resolved photo-emission spectroscopy (ARPES) shows reconstruction of the energy bands upon entering into the SDW state ${ }^{10-13}$. Some ARPES studies show an energy gap ${ }^{10,11}$, while others do not show any gap 12,13 in the SDW state. However, optical conductivity ${ }^{14}-17$, quantum oscillation ${ }^{18,19}$ and scanning tunneling microscopy and spectroscopy (STM/S) ${ }^{20}$ studies show an energy gap below the SDW transition. Parts of the Fermi surface disappear due to the nesting of the hole and electron bands at the Fermi energy in the SDW state and this leads to a reduction in DOS in certain energy range. In pnictides, both SDW and SC originate from the same Fe d-bands. This causes a competition between them over sharing of electronic states common to both gaps resulting in to an anti-correlation between the strengths of the SDW and SC order ${ }^{21}-23$.

We present STM/S study of in situ cleaved $\mathrm{SrFe}_{2} \mathrm{As}_{2}$ single crystals between $292 \mathrm{~K}$ and $6 \mathrm{~K}$. Resistivity measurement shows SDW transition at $\mathrm{T}_{S D W}=206 \mathrm{~K}$ in all crystals and $\mathrm{SC}$ transition at $\mathrm{T}_{c}=21 \mathrm{~K}$ in some of these undoped crystals. However, no diamagnetic behavior, expected in SC phase, is found. Tunneling spectra show a partial energy-gap below $\mathrm{T}_{S D W}$, which becomes more pronounced with cooling but the width of the gap starts reducing near $\mathrm{T}_{C}$. We interpret this weakening of the SDW order at low temperatures as a precursor effect to SC. This could imply an intrinsic propensity of this composition to becoming a SC resulting in to facile nucleation of SC near defects.

Single crystals of $\mathrm{SrFe}_{2} \mathrm{As}_{2}$ were grown by the high temperature solution growth method using Sn-flux un-

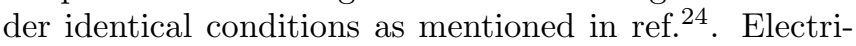
cal resistivity measurements were done using a standard four-probe method in a closed cycle refrigerator system. DC magnetization was measured using a vibrating sample magnetometer (VSM). STM/S studies were done in a cryogenic vacuum system using a homemade variable temperature STM system on in situ cleaved single crystals between 6 and $292 \mathrm{~K}$ temperature. Standard acmodulation technique was used for STS measurements with a modulation amplitude of $5 \mathrm{mV}$ and frequency 2731 $\mathrm{Hz}$. The in situ cleaving was done at room temperature and at $3 \times 10^{-6}$ mbar pressure before transferring the crystal to the STM head at low temperature. From tunnel spectra at different locations over $2 \times 2 \mu \mathrm{m}^{2}$ area and conductance maps we found the surface to be electronically homogeneous. Thus we plot spatial average of more than hundred spectra at a particular temperature. Similar temperature dependent spectra on several pieces of crystals were observed. We plot normalized differential conductance, i.e. $(\mathrm{dI} / \mathrm{dV}) /(\overline{I / V})$ to sharpen the spectral features where

$$
\overline{I / V}=\int_{-\infty}^{\infty} \frac{I\left(V^{\prime}\right)}{V^{\prime}} \exp \left(\frac{-\left|V^{\prime}-V\right|}{\Delta V}\right) d V^{\prime}
$$

\footnotetext{
a) adatta@iitk.ac.in
} 


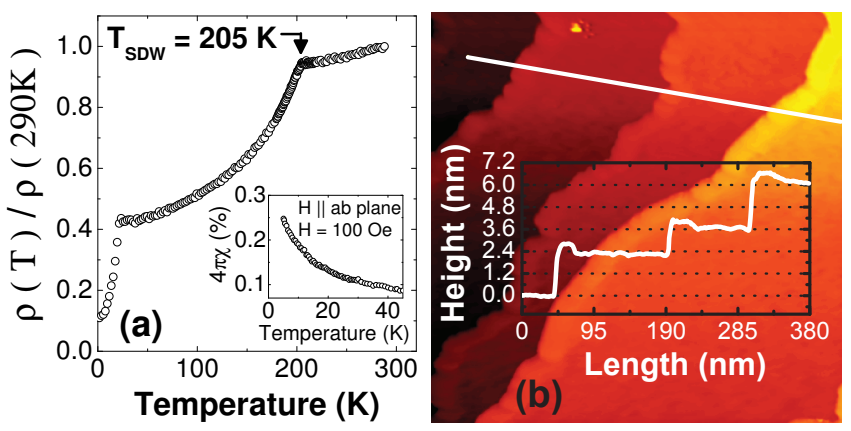

FIG. 1. (Color online) (a) Temperature dependence of the in-plane electrical resistivity for $\mathrm{SrFe}_{2} \mathrm{As}_{2}$ single crystal. Inset shows the temperature dependence of the zero field cooling (ZFC) magnetic susceptibility for $\mathrm{SrFe}_{2} \mathrm{As}_{2}$ single crystal in 100 Oe. (b) STM topographic image of in situ cleaved $\mathrm{SrFe}_{2} \mathrm{As}_{2}$ single crystal of area $414 \times 441 \mathrm{~nm}^{2}$ at $209.5 \mathrm{~K}$. Image was taken with $100 \mathrm{pA}$ tunnel current and $400 \mathrm{mV}$ bias. Inset shows the topographic profile along the marked line.

We have chosen $\Delta \mathrm{V}$ as $10 \mathrm{mV}$. Feenstra et al.$^{25}$ originally used this technique to study the energy gap of semiconductors by STM. Temperature also smears the spectral features of width less than a few $\mathrm{k}_{B} \mathrm{~T}$. This normalization procedure sharpens the features that are not thermally smeared by finite temperature.

The in-plane resistivity of $\mathrm{SrFe}_{2} \mathrm{As}_{2}$ crystals is plotted in Fig. 1(a) as a function of temperature. The resistivity decreases slowly with decreasing temperature below room temperature with a sharp kink at around $205 \mathrm{~K}$ due to an SDW transition ${ }^{8}$. Resistivity decreases rapidly as the temperature goes down further. The SDW transition opens a gap leading to some loss in density of states (DOS) at the Fermi energy leading to a sharp change in resistivity behavior. However, rapid decrease of resistivity with cooling implies only a partial gap and a finite DOS at $\mathrm{E}_{F}$ in the SDW state. Near $21 \mathrm{~K}$, there is sharp drop in resistivity, which we attribute to superconductivity. Such superconducting behavior is usually not seen in undoped pnictides. Saha et al. ${ }^{26}$ reported similar behavior together with a diamagnetism below $21 \mathrm{~K}$ in some of the as-grown $\mathrm{SrFe}_{2} \mathrm{As}_{2}$ crystals confirming the superconductivity. They attributed this superconductivity to the crystallographic strain. The magnetic susceptibility of one of our crystal as plotted in the inset of Fig. 11(a) does not show any diamagnetic behavior below $21 \mathrm{~K}$. Moreover, the resistivity never goes to zero down to 1.5 $K$. This implies that a very small portion of the crystal becomes superconductor below $21 \mathrm{~K}$, which may arise due to SC near defects. Superconducting volume here is small but it is well connected so as to show a drop in resistance. It is possible that this $\mathrm{SC}$ portion segregates near the surface or edges.

The STM topographic image of $\mathrm{SrFe}_{2} \mathrm{As}_{2}$ single crystal at $209.5 \mathrm{~K}$ in Fig. 1 (b) shows step-terrace morphology. The line profile in the inset of Fig. 1(b) shows that the terraces are separated by monatomic steps of height 1.2

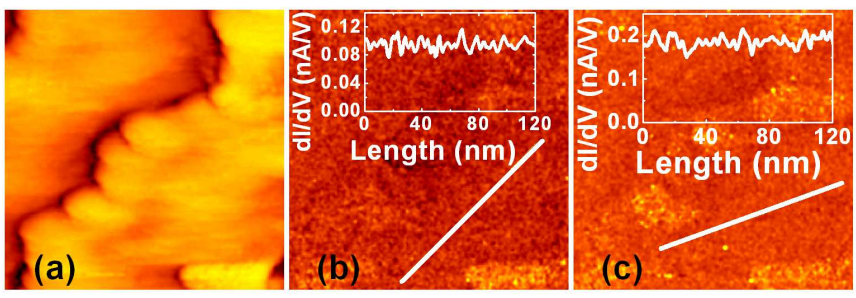

FIG. 2. (Color online) Simultaneously acquired STM topographic image and conductance maps of in situ cleaved $\mathrm{SrFe}_{2} \mathrm{As}_{2}$ single crystal at $178.5 \mathrm{~K}$ of area $190 \times 190 \mathrm{~nm}^{2}$. Images were taken with a tunnel junction of $100 \mathrm{pA}$ tunnel current and $400 \mathrm{mV}$ bias. (a) Topographic image. (b) Zerobias conductance map. (c) conductance map at $150 \mathrm{mV}$. Insets of (b) and (c) show the $\mathrm{dI} / \mathrm{dV}$ variations along the marked line in (b) and (c) respectively.

$( \pm 0.1) \mathrm{nm}$. The rms roughness over a terrace is less than $0.1 \mathrm{~nm}$. Similar step-terrace morphology is observed at all studied temperatures, indicating good quality of the cleaved surface. Fig. 2 shows the simultaneously acquired topographic image and conductance maps at $178.5 \mathrm{~K}$. Conductance maps were taken at two different bias voltages: 0 and $150 \mathrm{mV} . \mathrm{dI} / \mathrm{dV}$ along the marked lines, in the conductance maps as plotted in the insets of the respective maps, show very little variation. Similar homogeneous conductance maps were observed at other studied temperature.

Fig. 3(a) shows the temperature dependent spatially averaged tunnel spectra. Three representative I-V spectra at three different studied temperatures are also shown in Fig. 3(c). Each plotted conductance spectrum at a particular temperature is a spatial average of about 300 spectra taken over $2 \times 2 \mu \mathrm{m}^{2}$ area. The low temperature tunnel spectra show a dip near zero bias indicating the presence of a gap, while that at higher temperatures has only a noticeable curvature. Normalized spectra are shown in figure Fig. 3(b). The normalized spectrum at room temperature does not shows any depression near the $\mathrm{E}_{F}$. But as the temperature goes down, a broad depression arises in the normalized spectra near $\mathrm{E}_{F}$. The broad depression becomes more pronounced as the temperature goes below $\mathrm{T}_{S D W}$. We attribute this to the opening of a gap at $\mathrm{E}_{F}$ upon entering the SDW state. The slope of the I-V curve at zero bias which is proportional to the DOS at the $\mathrm{E}_{F}$, is less in the SDW phase at $6 \mathrm{~K}$ as compared to the paramagnetic phase at $292.1 \mathrm{~K}$ (see the Fig. 3(c)). The slope at zero-bias is non-zero at $6 \mathrm{~K}$. Thus the gap is not fully open in the SDW phase. Raw spectra taken at different locations over an area of $2 \times 2 \mu \mathrm{m}^{2}$ on the cleaved surface at two extreme temperatures 6 and $292.1 \mathrm{~K}$ are shown in the Fig. 4. Spectra at different locations have very small variations at both the temperatures and similar kind of local spectra were also observed at all the studied temperatures.

Normalized spectra show a partial gap in the SDW state. With cooling, the gap becomes more pronounced with a gap width of $\sim 98 \mathrm{mV}$ at $78 \mathrm{~K}$. The gap width 

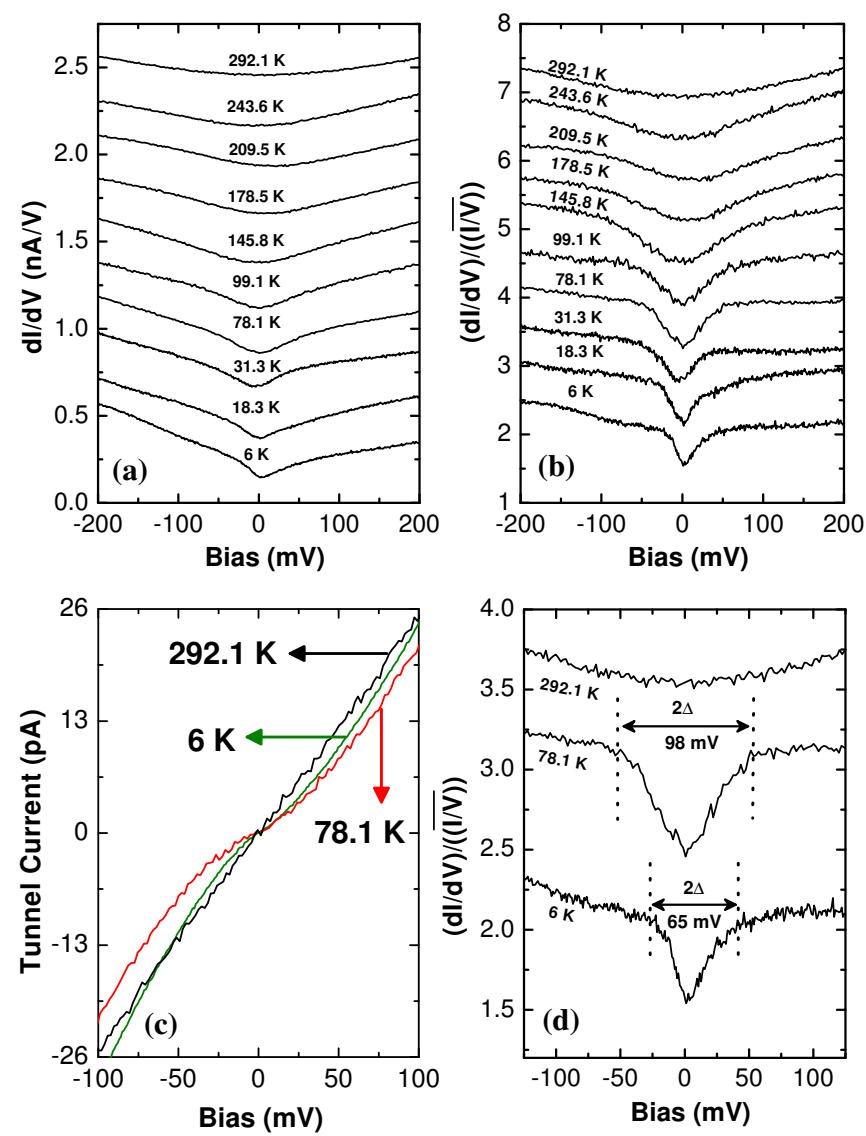

FIG. 3. (Color online) (a) dI/dV Vs. V at $292.1 \mathrm{~K}-6 \mathrm{~K}$, (b) normalized dI/dV Vs. V at $292.1 \mathrm{~K}-6 \mathrm{~K}$, (c) Voltage dependent tunnel current at $292.1 \mathrm{~K}, 78.1 \mathrm{~K}$ and $6.0 \mathrm{~K}$ and (d) Expended view of normalized $\mathrm{dI} / \mathrm{dV}$ Vs. V at $292.1 \mathrm{~K}, 78.1$ $\mathrm{K}$ and $6.0 \mathrm{~K}$ of in situ cleaved $\mathrm{SrFe}_{2} \mathrm{As}_{2}$ single crystals. The tunnel current and spectra were taken with a junction bias of $300 \mathrm{mV}$ and a tunnel current of $100 \mathrm{pA}$. Consecutive spectra in (a), (b) and (d) have been shifted uniformly upwards for clarity.

is estimated from the separation between the two edges in the normalized spectra, where the depression starts to appear as marked by the dotted lines in Fig. 3. (d). But with further cooling, the spectra from $31 \mathrm{~K}-6 \mathrm{~K}$ show that the gap width reduces significantly without change in depth. At $6 \mathrm{~K}$, the estimated gap width is $\sim 65 \mathrm{mV}$. Similar trend is also clearly visible in the $\mathrm{dI} / \mathrm{dV}-\mathrm{V}$ plot. Normalization procedure just sharpens the gap feature without adding (loosing) any extra (existing) feature. At any finite temperature $\mathrm{T}$, the spectral features are smeared out with a few $\mathrm{k}_{B} \mathrm{~T}\left(\sim 3.5 \mathrm{k}_{B} \mathrm{~T}\right)$ spread. Thus at low temperatures the smearing induced broadening of the SDW gap will be less, which would also make the gap appear smaller but deeper. The reduction in gap from $78 \mathrm{~K}$ to $6 \mathrm{~K}$ is $\sim 33 \mathrm{meV}$ which is $\sim 5 \mathrm{k}_{B} \mathrm{~T}$ but the depth of the gap feature is almost unchanged. So the reduction in the gap width cannot be attributed to reduction in thermal smearing.

It is possible that the surface of the studied crystals
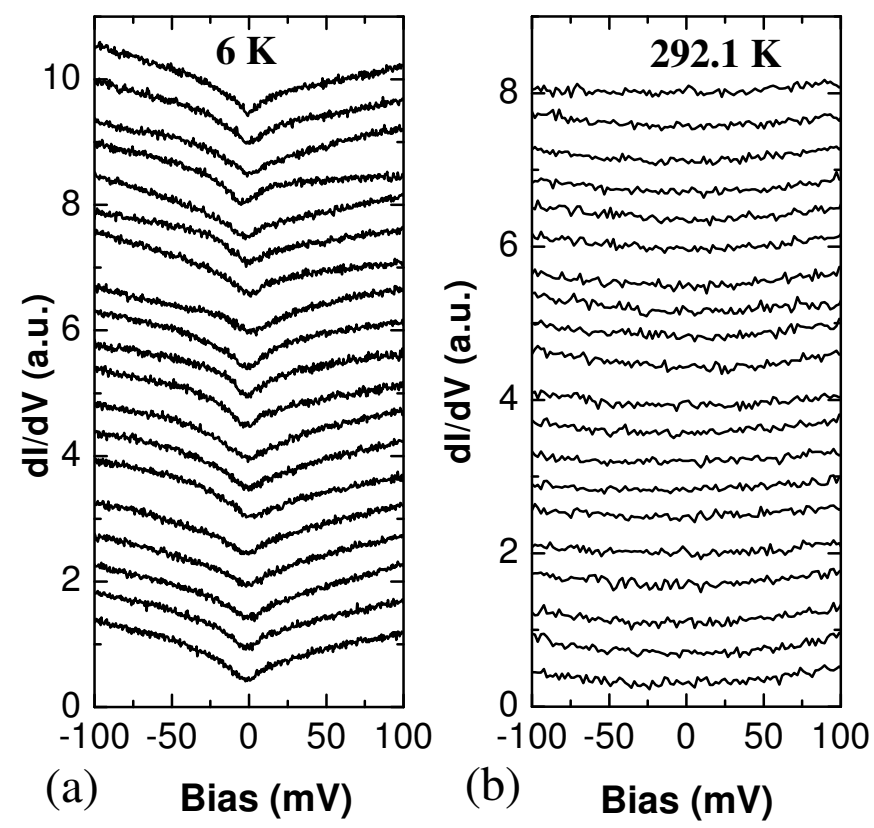

FIG. 4. dI/dV Vs. V spectra taken at different locations over an area of $2 \times 2 \mu \mathrm{m}^{2}$ of in situ cleaved $\mathrm{SrFe}_{2} \mathrm{As}_{2}$ single crystal at (a) $6.0 \mathrm{~K}$ and (b) $292.1 \mathrm{~K}$. Consecutive spectra have been shifted uniformly upwards for clarity.

differs from the bulk due to the room temperature vacuum cleaving or otherwise 27 . We have observed similar spectra over large area spanning $2 \times 2 \mu \mathrm{m}^{2}$ of cleaved surface and on several different pieces of crystals cleaved similarly. We can not make any comment on the surface reconstruction or termination plane of the studied surface, as we do not have atomically resolved STM images. One STM/S study on room temperature cleaved $\mathrm{BaFe}_{1.86} \mathrm{Co}_{0.14} \mathrm{As}_{2}$ showed that termination plane topographic disorder has very little effect on the low-lying electronic states of these systems ${ }^{28}$. A hard X-ray photoemission also supports this finding 29 . Thus we believe that the observed spectral features near Fermi energy do reflect intrinsic property of these crystals.

Optical spectroscopy on $\mathrm{SrFe}_{2} \mathrm{As}_{2}{ }^{14,15}, \mathrm{CaFe}_{2} \mathrm{As}_{2}{ }^{15}$ and $\mathrm{BaFe}_{2} \mathrm{As}_{2} 14,15$ shows presence of two gaps. In $\mathrm{SrFe}_{2} \mathrm{As}_{2}$, Hu et al. ${ }^{14}$ identified two gaps with peaks at $500 \mathrm{~cm}^{-1}$ and $1500 \mathrm{~cm}^{-1}$ which correspond to $62.04 \mathrm{meV}$ and $168.75 \mathrm{meV}$ respectively. Charnukha et al. $\frac{15}{}$ also reported two gaps for $\mathrm{SrFe}_{2} \mathrm{As}_{2}$, but with slightly lower values (380 and $1215 \mathrm{~cm}^{-1}$ ). Our tunneling spectra show only one gap and it's magnitude almost matches with the smaller gap observed by $\mathrm{Hu}$ et al. $\stackrel{14}{ }$. A pseudogap of $500 \mathrm{~cm}^{-1}$ without any sign of gap at higher energy is observed in one optical study ${ }^{16}$ on $\mathrm{BaFe}_{2} \mathrm{As}_{2}$. Another optical investigation ${ }^{17}$ on $\mathrm{EuFe}_{2} \mathrm{As}_{2}$ also doesn't show the second gap. Our earlier tunneling study ${ }^{20}$ on $\mathrm{EuFe}_{2} \mathrm{As}_{2}$ also showed a single gap like spectra.

In 122-pnictides the SDW and SC phases coexist over a finite doping range ${ }^{2-4}$ and they can coexist both homogeneously and inhomogeneously as reported by several 
groups $^{21-23,30,31}$. The STM/S study by Cai et al..$^{23}$ in underdoped $\mathrm{NaFe}_{1-x} \mathrm{Co}_{x} \mathrm{As}_{2}$ showed an anti-correlation between the strength of the SDW and SC order, indicating a competition between the two. Nevertheless, the $\mathrm{SC}$ order clearly anti-correlates, particularly in the underdoped regime, with the SDW in the sense that SC appears when SDW declines. Reduction of SDW gap at low temperature in our results could be a precursor effect to an upcoming SC order, which does not quite happen in the bulk of this undoped crystal. Although resistivity shows a SC transition near $21 \mathrm{~K}$ in some of our crystals similar to an earlier report ${ }^{26}$ where $\mathrm{SC}$ was attributed to strain related to defects. Magnetic fluctuations are believed to play an important role in SC of pnictides but long range magnetic order does not favor SC. The weakening of the SDW order at low temperatures will certainly make it easier for SC to nucleate, at least near defects.

In conclusion, our variable temperature STM/S study shows a partial energy gap due to SDW order below 200 $\mathrm{K}$, which starts becoming weaker at low temperatures below about 30K. The resistivity of some of the crystals shows a partial SC transition at $21 \mathrm{~K}$ with negligible $\mathrm{SC}$ fraction inferred from magnetic susceptibility. The weakening of SDW order at low temperatures could be a precursor to SC order and indicates an intrinsic inclination of these crystals to becoming a superconductor resulting into facile nucleation of $\mathrm{SC}$ near defects as seen in resistivity.

We thank Sourabh Barua for his help in the resistivity measurement. Anirban acknowledges financial support from the CSIR of the Government of India. A.K.G. acknowledges a research grant from the CSIR of the Government of India. We would like to acknowledge Avinash Singh and Sayandip Ghosh for helpful discussions.

\footnotetext{
${ }^{1}$ Y. Kamihara, T. Watanabe, M. Hirano, and H. Hosono, J. Am. Chem. Soc. 130, 3296 (2008).

${ }^{2}$ D. C. Johnston, Adv. Phys. 59, 803 (2010).

${ }^{3}$ G. R. Stewart, Rev. Mod. Phys. 83, 1589 (2011).

${ }^{4}$ A. A. Kordyuk, Low Temp. Phys. 38, 888 (2012).

${ }^{5}$ C. W. Chu and B. Lorenz, Physica C 469, 385 (2009).
}

${ }^{6}$ M. Rotter, M. Tegel, D. Johrend, I. Schellenberg, W. Hermes, and R. Pöttgen, Phys. Rev. B 78, 020503(R) (2008).

${ }^{7}$ F. Ronning, T. Klimczuk, E. D. Bauer, H. Volz and J. D. Thompson, J. Phys.: Condens. Matter 20, 322201 (2008).

${ }^{8}$ C. Krellner, N. Caroca-Canales, A. Jesche, H. Rosner, A. Ormeci, and C. Geibel, Phys. Rev. B 78, 100504(R) (2008).

${ }^{9}$ H. S. Jeevan, Z. Hossain, D. Kasinathan, H. Rosner, C. Geibel, and P. Gegenwart, Phys. Rev. B 78, 052502 (2008).

${ }^{10}$ L. X. Yang et al., Phys. Rev. Lett. 102, 0107002 (2009).

${ }^{11}$ S. de Jong et al., Europhys. Lett. 89, 27007 (2010).

${ }^{12}$ Y. Zhang et al., Phys. Rev. Lett. 102, 127003 (2009).

${ }^{13}$ C. Liu et al., Phys. Rev. Lett. 101, 177005 (2008).

${ }^{14}$ W. Z. Hu, J. Dong, G. Li, Z. Li, P. Zheng, G. F. Chen, J. L. Luo, and N. L. Wang, Phys. Rev. Lett. 101, 257005 (2008).

${ }^{15}$ A. Charnukha, D. Pröpper, T. I. Larkin, D. L. Sun, Z. W. Li, C. T. Lin, T. Wolf, B. Keimer, and A. V. Boris, Phys. Rev. B 88, 184511 (2013).

${ }^{16}$ F. Pfuner, J. G. Analytis, J.-H. Chu, I. R. Fisher, and L. Degiorgi, Eur. Phys. J. B 67, 513 (2009).

${ }^{17}$ D. Wu et. al., Phys. Rev. B 79, 155103 (2009).

${ }^{18}$ S. E. Sebastian, J. Gillett, N. Harrison, P. H. C. Lau, D. J. Singh, C. H. Mielke and G. G. Lonzarich, J. Phys.: Condens. Matter 20, 422203 (2008).

${ }^{19}$ J. G. Analytis, R. D. McDonald, J.-H.Chu, S. C. Riggs, A. F. Bangura, C. Kucharczyk, M. Johannes, and I. R. Fisher, Phys. Rev. B 80, 064507 (2009).

${ }^{20}$ A. Dutta, Anupam, Z. Hossain and A. K. Gupta, J. Phys.: Condens. Matter 25, 375602 (2013).

${ }^{21}$ D. K. Pratt, W. Tian, A. Kreyssig, J. L. Zarestky, S. Nandi, N. Ni, S. L. Budko, P. C. Canfield, A. I. Goldman, and R. J. McQueeney, Phys. Rev. Lett. 103, 087001 (2009).

${ }^{22}$ Rafael M. Fernandes, Phys. Rev. B 81, 140501(R) (2010).

${ }^{23}$ P. Cai, X. Zhou, W. Ruan, A. Wang, X. Chen, D-H. Lee and Y. Wang, Nat. Commun. 4, 1596 (2013).

${ }^{24}$ N. Kumar, R. Nagalakshmi, R. Kulkarni, P. L. Paulose, A. K. Nigam, S. K. Dhar, and A. Thamizhavel, Phys. Rev. B 79, 012504 (2009).

${ }^{25}$ R. M. Feenstra, Phys. Rev. B 50, 4561 (1994).

${ }^{26}$ S. R. Saha, N. P. Butch, K. Kirshenbaum, Johnpierre Paglione and P.Y. Zavalij, Phys. Rev. Lett. 103, 037005 (2009).

${ }^{27}$ Jennifer E Hoffman, Rep. Prog. Phys. 74, 124513 (2011).

${ }^{28}$ F. Massee, Y. Huang, R. Huisman, S. de Jong, J. B. Goedkoop, and M. S. Golden, Phys. Rev. B 79 (2009) 220517(R).

${ }^{29}$ S. de Jong, Y. Huang, R. Huisman, F. Massee, S. Thirupathaiah, M. Gorgoi, F. Schaefers, R. Follath, J. B. Goedkoop, and M. S. Golden, Phys. Rev. B 79 (2009) 115125.

${ }^{30}$ M.-H. Julien et al., Europhys. Lett. 87, 37001 (2009).

${ }^{31}$ J. T. Park et al. Phys. Rev. Lett. 102, 117006 (2009). 